\title{
When to Bite the Bullet? - A Study of Optimal Strategies for Reducing Global Warming
}

\author{
$\underline{\text { X. Luo, P. V. Shevchenko }}{ }^{\text {a }}$ \\ ${ }^{a}$ CSIRO Computational Informatics, Sydney NSW Australia \\ Email: Xiaolin.Luo@csiro.au
}

\begin{abstract}
This work is based on the framework proposed by Conrad (1997) to determine the optimal timing of an investment or policy to slow global warming. While Conrad formulated the problem as a stopping rule option pricing model, we treat the policy decision by considering the total damage function that enables us to make some interesting extensions to the original formulation. We show that Conrad's framework is equivalent to minmization of the expected value of the damage function under the stochastic optimal stopping rule. We extend Conrad's model by allowing for policy cost to grow with time. In addition to closed form solution, we also perform Monte Carlo simulations to find the distribution for the total damage and show that at higher quantiles the damage may become too large and so is the risk on the global economy. We also show that the decision to take action largely depends on the cost of the action. For example, in the case of model parameters calibrated as in Conrad (1997) with a constant cost, there is a rather long wait before the action is expected to be taken, but if the cost increases with the same rate as the global economy growth, then action has to be taken immediately to minimize the damage.
\end{abstract}

Keywords: Stochastic optimal stopping rule, global warming, Monte Carlo simulations 


\section{INTRODUCTION}

Over the last few decades, scientists have studied extensively the effect of global warming and optimal strategies to slow down the process to manage disaster risks. For example, see Nordhaus (1991), Nordhaus (1992), Nordhaus and Boyer (2000), Association of American Geographyers (2003), UNFCCC (2006), Nordhaus (2008), Greiner et al. (2010), Field et al. (2012). Some of these models establish the close linkage between global economy growth and the damage by global warming and support the view that policy actions against global warming are urgently needed.

In this paper, we study the global warming model of Conrad (1997) and suggest optimal timing for the policy by considering the total damage function. The global temperature is assumed to be drifting upward following a Brownian motion. Investments to slow the global warming are called "bullets" and they are characterized by the reduction of both the drift rate and the standard deviation after the investments. If the action is taken at time $t=\tau$ which reduces the drift from $\mu_{1}$ to $\mu_{2}$ and the volatility from $\xi_{1}$ to $\xi_{2}$, the temperature process is assumed to be

$$
d C_{t}=\mu(t) d t+\xi(t) d z, \quad \mu(t)=\mu_{1} I_{t<\tau}+\mu_{2} I_{t \geq \tau}, \quad \xi(t)=\xi_{1} I_{t<\tau}+\xi_{2} I_{t \geq \tau},
$$

where $C_{t}$ is the mean global temperature at time $t, \mu(t)$ is the drift, $\xi(t)$ is the volatility, $d z$ is the increment of the standard Wiener process and $I_{\{.\}}$is the indicator symbol that equals 1 if the condition in $\{$.$\} is true and 0$ otherwise. The damage rate from global warming is assumed to be the convex function

$$
S_{t}=S_{0} e^{\gamma\left(C_{t}-C_{0}\right)}
$$

where $S_{t}$ is the damage rate in billions of dollars per annum, $\gamma$ is a positive parameter, $C_{0}$ is the reference temperature and $S_{0}$ is the corresponding damage rate. The original model was calibrated using time series data for temperature anomalies and estimates of the damage from the global warming and the cost of the investments. In this study we extend Conrad's model by allowing for policy cost to grow with time. In addition to closed form solution for the optimal timing of the policy, we also perform Monte Carlo simulations to find the distribution for the possible total damage.

\section{STOPPING-RULE MODEL AND SOLUTION}

Applying Ito's Lemma to function (2) with process (1), one can show that the damage rate $S_{t}$ from global warming follows the geometric Brownian motion with dirft $\alpha(t)$ and volatility $\sigma(t)$

$$
d S_{t}=\alpha(t) S_{t} d t+\sigma(t) S_{t} d z, \quad \alpha(t)=\alpha_{1} I_{t<\tau}+\alpha_{2} I_{t \geq \tau}, \quad \sigma(t)=\sigma_{1} I_{t<\tau}+\sigma_{2} I_{t \geq \tau},
$$

where $\alpha_{1}=\mu_{1} \gamma+\left(\xi_{1} \gamma\right)^{2} / 2$ and $\sigma_{1}=\xi_{1} \gamma$ are the drift and volatility before the policy is introduced, $\alpha_{2}=\mu_{2} \gamma+\left(\xi_{2} \gamma\right)^{2} / 2<\alpha_{1}$ and $\sigma_{2}=\xi_{2} \gamma<\sigma_{1}$ are the drift and volatility after the policy, $d z$ is the increment of a standard Wiener proces. We assume that the cost of the policy changes in time as $K_{t}=K \exp (q \times t)$ and it is paid only once when the policy is introduced at time $t=\tau$. It is important to note that Conrad (1997) assumes that the cost of policy is time independent. It is further assumed that the global economy grows at a rate $r>\alpha_{1}$.

From (3) it follows that the damage rate is log-normally distributed, thus the expected value of the damage at future time $T$, given damage rate $S_{t}$ at time $t$, is $E_{t}\left[S_{T}\right]=S_{t} \exp \left(\int_{t}^{T} \alpha(x) d x\right)$. Hereafter, notation $E_{t}[\cdot]$ denotes expectation conditional on information available at time $t$. Thus the present value at time $t$ of the future total expected damage if the policy is never introduced can be easily calculated as

$$
d_{1}\left(S_{t}\right)=E_{t}\left[\int_{t}^{\infty} S_{t^{\prime}} e^{-r\left(t^{\prime}-t\right)} d t^{\prime}\right]=S_{t} \int_{t}^{\infty} e^{\alpha_{1}\left(t^{\prime}-t\right)} e^{-r\left(t^{\prime}-t\right)} d t^{\prime}=\frac{S_{t}}{\left(r-\alpha_{1}\right)} .
$$

Similarly, if the policy is introduced at time $t$, the total discounted expected future damage plus the policy cost is given by

$$
d_{2}\left(S_{t}\right)=E_{t}\left[\int_{t}^{\infty} S_{t^{\prime}} e^{-r\left(t^{\prime}-t\right)} d t^{\prime}\right]+K_{t}=\frac{S_{t}}{\left(r-\alpha_{2}\right)}+K_{t} .
$$

Consider the actual total damage function represented by random variable

$$
D(t, \tau)=\int_{t}^{\tau} S_{x} e^{-r(x-t)} d x+\int_{\tau}^{\infty} S_{x} e^{-r(x-t)} d x+K_{\tau} e^{-r(\tau-t)} .
$$


Here, $\tau$ is stochastic stopping time, i.e. different realizations will have different $\tau$. The expected total damage under the stopping rule $\tau$ for damage rate $S$ at time $t$ is

$$
V(S, t)=E_{t}[D(t, \tau)]
$$

Typically, one defines an optimal stopping rule $\tau^{*}$ as the one that minimizes expected value of $D(t, \tau)$, i.e. the expected total damage under the optimal stopping rule is

$$
V^{*}(S, t)=\min _{\tau} E_{t}[D(t, \tau)]=E_{t}\left[D\left(t, \tau^{*}\right)\right] .
$$

We can solve this problem by formulating partial differential equation (PDE) for $V(S, t)$; for detailed discussion of PDE approach for valuation of projects and investment rules, see Dixit and Pindyck (1994).

It is interesting to consider the deterministic case when the volatility $\sigma_{1}=\sigma_{2}=0$ in (3). In this case $S_{t}=S_{0} e^{\alpha_{1} t}$ when $t<\tau$ and $S_{t}=S_{0} e^{\alpha_{1} \tau+\alpha_{2}(t-\tau)}$ when $t \geq \tau$. Minimizing the deterministic function $D(t, \tau)$ with respect to $\tau$ we obtain the optimal time $\tau^{*}$ and the corresponding critical damage rate $S^{*}$

$$
\tau^{*}=\frac{1}{\alpha_{1}-q} \ln \left(\frac{K(r-q)\left(\alpha_{2}-r\right)}{S_{0}\left(\alpha_{2}-\alpha_{1}\right)}\right), \quad S^{*}=S_{0}\left(\frac{K(r-q)\left(\alpha_{2}-r\right)}{S_{0}\left(\alpha_{2}-\alpha_{1}\right)}\right)^{\frac{\alpha_{1}}{\alpha_{1}-q}}
$$

if $q<\alpha_{1}$. If the process is stochastic instead of deterministic, there is some value in waiting somewhat longer than the deterministic case, as will be shown later with an actual example.

Now for the stochastic process consider the region where the policy is not introduced, i.e. the transition density function $f\left(S^{\prime}, t^{\prime} \mid S, t\right)$ for the damage rate stochastic process (3) satisfies the backward Kolmogorov equation

$$
\frac{\partial f\left(S^{\prime}, t^{\prime} \mid S, t\right)}{\partial t}+\alpha_{1} S \frac{\partial f\left(S^{\prime}, t^{\prime} \mid S, t\right)}{\partial S}+\frac{1}{2} \sigma_{1}^{2} S^{2} \frac{\partial^{2} f\left(S^{\prime}, t^{\prime} \mid S, t\right)}{\partial S^{2}}=0
$$

for transition densities and Kolmogorov equations corresponding to Wiener processes, see Cox and Miller (1965). Multiplying each term by $S^{\prime} e^{-r\left(t^{\prime}-t\right)}$, integrating from $t$ to $\infty$ and taking expectation yields

$$
\frac{\partial V(S, t)}{\partial t}+\alpha_{1} S \frac{\partial V(S, t)}{\partial S}+\frac{1}{2} \sigma_{1}^{2} S^{2} \frac{\partial^{2} V(S, t)}{\partial S^{2}}-r V(S, t)+S=0
$$

subject to the condition $V(S, t) \leq d_{2}(S)+K_{t}$, where the equality applies on the boundary $S=H_{t}$ when policy is introduced.

Note that the terms $r V$ and $S$ appear because we move $e^{-r\left(t^{\prime}-t\right)}$ and integration $\int_{t}^{\infty}$ under the derivative $\partial f / \partial t$. Also note that we do not start the policy if $V(S, t)<d_{2}(S(t))$ and introduce the policy otherwise.

The policy cost is time dependent $K_{t}=K \exp (q \times t)$, thus the problem is time non-homogenous. Assume that the policy is introduced when damage rate $S_{t}$ breaches the level $H_{t}=H \exp (q \times t)$. To reduce the problem to time homogeneous and solve PDE, consider a new variable $Y=S \exp (-q \times t)$. The PDE for $Q(Y, t) \equiv V(S, t)$ will be the same as (11) except the change of $\alpha_{1}$ to $\alpha_{1}-q$. Substituting solution in the form $Q(Y, t)=\widetilde{Q}(Y) \exp (q \times t)$ leads to ODE for $\widetilde{Q}(Y)$

$$
\left(\alpha_{1}-q\right) Y \frac{d \widetilde{Q}(Y)}{d Y}+\frac{\sigma_{1}^{2}}{2} Y^{2} \frac{d^{2} \widetilde{Q}(Y)}{d Y^{2}}-(r-q) \widetilde{Q}(Y)+Y=0
$$

subject to the condition $\widetilde{Q}(Y) \leq \frac{Y}{\left(r-\alpha_{2}\right)}+K$, where the equality applies to the boundary at $Y=H$. Equation (12) is a non-homogeneous, second-order differential equation. The homogeneous part has the well-known solution $\widetilde{Q}(Y)=\xi Y^{\tilde{\epsilon}}+\eta Y^{\epsilon}$, where $\tilde{\epsilon}$ and $\epsilon$ are given by

$$
\begin{aligned}
\tilde{\epsilon} & =\left(1 / 2-\left(\alpha_{1}-q\right) / \sigma_{1}^{2}\right)-\sqrt{\left(1 / 2-\left(\alpha_{1}-q\right) / \sigma_{1}^{2}\right)^{2}+2(r-q) / \sigma_{1}^{2}} \\
\epsilon & =\left(1 / 2-\left(\alpha_{1}-q\right) / \sigma_{1}^{2}\right)+\sqrt{\left(1 / 2-\left(\alpha_{1}-q\right) / \sigma_{1}^{2}\right)^{2}+2(r-q) / \sigma_{1}^{2}}
\end{aligned}
$$

and constants $\xi, \eta$ can be found from the boundary conditions. One of the boundary conditions is $\widetilde{Q}(0)=0$. It can be shown that if $q<r$ then $\tilde{\epsilon}<0$. Thus the conditions $\widetilde{Q}(0)=0$ and $\tilde{\epsilon}<0$ lead to $\xi=0$ and solution 
simplifies to $\widetilde{Q}(Y)=\eta Y^{\epsilon}$. On the other hand, if $q \geq r, \tilde{\epsilon} \geq 0$, both terms $\xi Y^{\tilde{\epsilon}}$ and $\eta Y^{\epsilon}$ are retained in the solution. Here we only consider the solution for $q<r$.

A particular solution to equation (12) is simply $Y /\left(r-\alpha_{1}\right)$. Thus a general solution is obtained by adding the solution of the homogenuous portion to the particular solution yielding

$$
\widetilde{Q}(Y)=\eta Y^{\epsilon}+\frac{Y}{\left(r-\alpha_{1}\right)}
$$

The continuity conditions for the function on the boundary $Y=H$, i.e. $\widetilde{Q}(Y)=\frac{Y}{\left(r-\alpha_{2}\right)}+K$, gives

$$
\eta H^{\epsilon}+\frac{H}{\left(r-\alpha_{1}\right)}=\frac{H}{\left(r-\alpha_{2}\right)}+K
$$

that allows to identify constant $\eta$

$$
\eta=\frac{1}{H^{\epsilon-1}} \frac{\left(\alpha_{2}-\alpha_{1}\right)}{\left(r-\alpha_{1}\right)\left(r-\alpha_{2}\right)}+\frac{K}{H^{\epsilon}} .
$$

Thus the solution for total damage is $V(S, t)=\widetilde{Q}\left(S e^{-q t}\right) e^{q t}$, where

$$
\begin{aligned}
\widetilde{Q}(Y) & =\eta Y^{\epsilon}+\frac{Y}{\left(r-\alpha_{1}\right)}=\left(\frac{K}{H^{\epsilon}}-\frac{1}{H^{\epsilon-1}} \frac{\left(\alpha_{1}-\alpha_{2}\right)}{\left(r-\alpha_{1}\right)\left(r-\alpha_{2}\right)}\right) Y^{\epsilon}+\frac{Y}{\left(r-\alpha_{1}\right)} \\
& =\left(\frac{Y}{H}\right)^{\epsilon}\left(K-H \frac{\alpha_{1}-\alpha_{2}}{\left(r-\alpha_{1}\right)\left(r-\alpha_{2}\right)}\right)+\frac{Y}{\left(r-\alpha_{1}\right)} .
\end{aligned}
$$

Considering $V(S, t)$ as a function of boundary level $H$, we can find optimal level $H^{*}$, where $V(S, t)$ is minimized with respect to $H$ by solving

$$
\frac{\partial \widetilde{Q}(Y)}{\partial H}=Y^{\epsilon}\left(-\epsilon \frac{K}{H^{\epsilon+1}}+\frac{\epsilon-1}{H^{\epsilon}} \frac{\alpha_{1}-\alpha_{2}}{\left(r-\alpha_{1}\right)\left(r-\alpha_{2}\right)}\right)=0
$$

that gives

$$
H^{*}=\frac{\epsilon\left(r-\alpha_{1}\right)\left(r-\alpha_{2}\right) K}{(\epsilon-1)\left(\alpha_{1}-\alpha_{2}\right)} .
$$

We could obtain the same result if we would solve PDE (11) for optimal $V^{*}(S, t)$ directly; in that case we just had to impose continuity condition not just for the function but also for its 1 st derivative on the stopping boundary; for discussion about these boundary conditions, see Dixit and Pindyck (1994, pp. 130-132).

The corresponding optimal critical damage rate is $S^{*}=e^{q t} H^{*}$. The probability density of the hitting time $\tau$ for process $Y_{t}$ to breach level $H$ (i.e. $S_{t}$ to breach $H_{t}$ ) is given by

$$
g(\tau)=\frac{\ln \left(H / S_{0}\right)}{\sigma_{1} \tau \sqrt{2 \pi \tau}} \exp \left\{-\frac{\left[\ln \left(H / S_{0}\right)-\eta \tau\right]^{2}}{2 \sigma_{1}^{2} \tau}\right\}, \quad \eta=\alpha_{1}-q-\sigma_{1}^{2} / 2>0, \text { for } H>Y_{0}=S_{0} .
$$

The above density is only valid if $\eta>0$. From (21) we find the expected hitting time is

$$
\mathrm{E}[\tau]=\frac{\ln \left(H / S_{0}\right)}{\eta}
$$

In the case of time independent cost (i.e. $q=0$ ), the expected critical time (to breach the optimal critical level $\left.S^{*}\right)$ from the above equation reduces to $\left(C^{*}-C_{0}\right) / \mu_{1}$, where $C^{*}=C_{0}+\ln \left(S^{*} / S_{0}\right) / \gamma$ is the critical temperature corresponding to $S^{*}$. The solution obtained here for $q=0$ is equivalent to the solution in Conrad (1997). Finally, the solution to the stochastic problem also contains the deterministic case given in (9): by letting $\sigma_{1}=\sigma_{2} \rightarrow 0$, we find $\epsilon \rightarrow(r-q) /\left(\alpha_{1}-q\right)$ and the solution for $\tau^{*}$ and $S^{*}$ is identical to (9). 


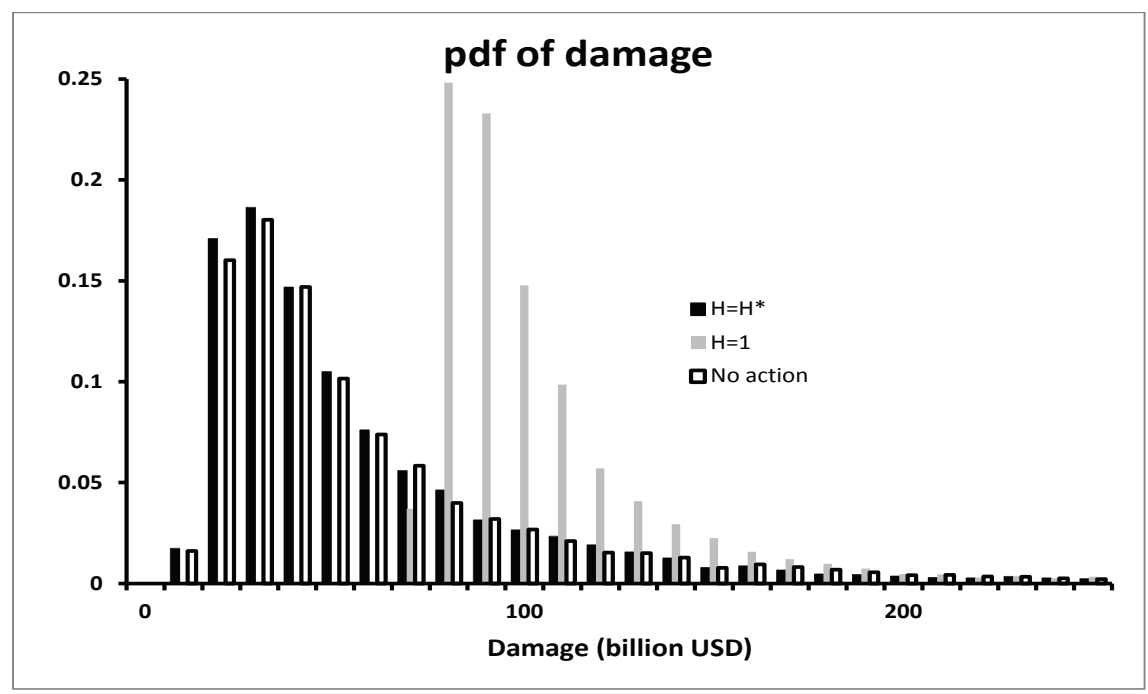

Figure 1. Probability density function (pdf) for the total damage. $H=1$ is for taking action immediately, $H=H^{*}$ is for optimal strategy and "No action" corresponds to never taking any action (business as usual).

\section{NUMERICAL RESULTS}

Here we analyse the distribution of total damage $D(t, \tau)$ given by (6) under the optimal and non optimal stopping rules. Monte Carlo simulation of $D(t, \tau)$ is simple: one has to simulate damage rate process $S(t)$ given by (3) with drift $\alpha_{1}$ until time $\tau$ where $S(t)$ breaches the critical level $H \exp (q \times t)$ and with drift $\alpha_{2}$ afterwards, and calculate $D(t, \tau)$ in (6). Repeating simulation many times allows us to find a distribution of $D(t, \tau)$.

The parameter values of $r=0.05, \alpha_{1}=0.03708657, \alpha_{2}=0.02758027, \sigma_{1}=\sigma_{2}=0.19012608$ are taken from Conrad (1997). For the base solution, we assume a constant cost, i.e. $q=0.0$, that corresponds to Conrad's work. The case of constant cost assumes the future value of the cost remains the same, which effectively make the present value of the cost getting cheaper at the same rate as the economy growth rate, a somewhat unrealistic assumption.

Typically, the solution to the stopping rule problem, or equivalently to the American option pricing problem, is in terms of expected value of payoff function. In the context of financial options, this expectation gives a fair value at which there are buyers and sellers willing to do the transaction, knowing that the associated risks can be fully hedged. If these kind of buying and selling are repeated many times, on average these transactions will give the buyers or sellers a fair return adjusted to the risks they take. However, to the question of when to take action to slow down global climate change, the answer based on average damage (expected value) is too risky and almost does not make sense. There is only one realization of reality and one chance for us to do it right. The risk to human being's survival is too great for us to rely on an average solution to manage the risk.

Indeed, the solution based on the expectations gives $\tau^{*}=122$ years as the expected optimal time to take action, a seemingly very long time. The optimal critical damage rate $S^{*}=$ USD 10.19 billion per annum at which the action should be taken to minimize the expected total damage. The corresponding critical temperature is $C^{*}=16.22$ degrees, assuming today's temperature is $C_{0}=15$ degrees. Using 100,000 Monte Carlo simulations, the expected total damage from this optimal strategy is about USD 61.6 billion with a numerical standard error of 0.83 billion due to finite number of simulations.

In comparison with the the above solution for the stochastic case, the corresponding solution for the deterministic case in (9) gives $\tau^{*}=53$ years and $S^{*}=$ USD 7.08 billion per annum, both are much smaller than the stochastic counterparts, demonstrating the value in waiting under uncertainty.

Instead of considering the expectations, a more sensible approach is by considering the entire distribution of the total damage. The Monte Carlo simulation allows us to obtain such a distribution. The pdf of total damage for the case of constant cost is shown in Figure 1. Given the distribution, higher quantiles can be 


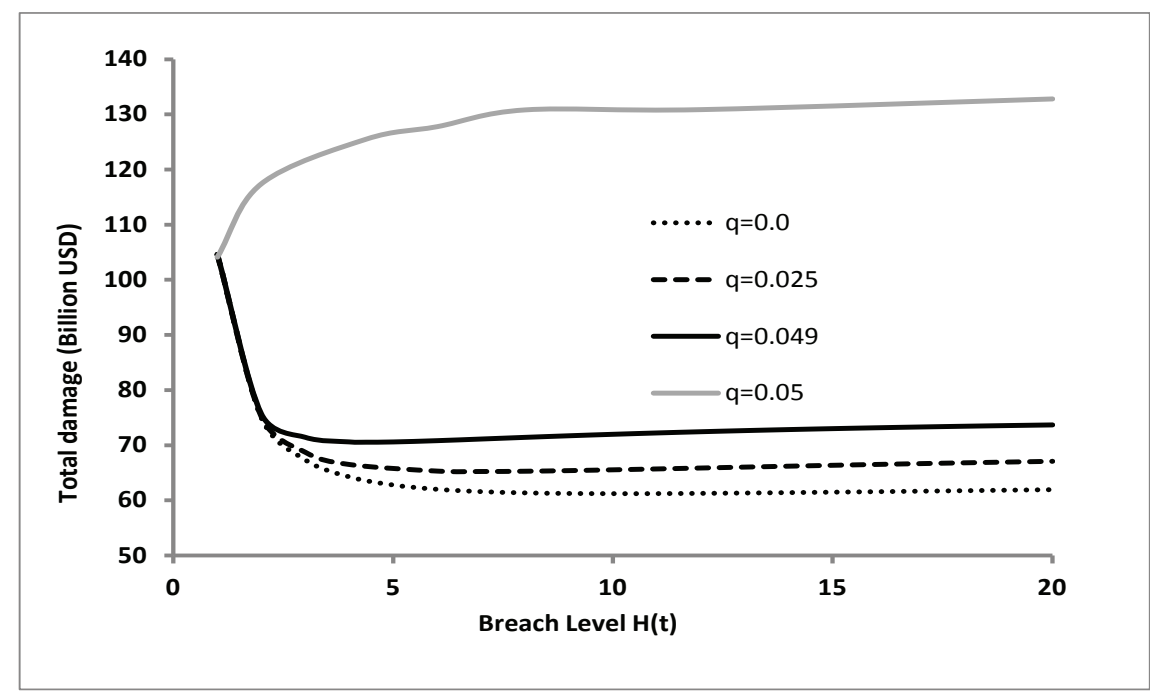

Figure 2. Damage curves for different cost increasing rate $q$. The curve for $q=0.05$ is from Monte Carlo simulation, and all others are analytical solutions.

Table 1. Quantiles of total damage (billion USD)

\begin{tabular}{cccc}
\hline Cases & Mean & Median & 0.9 quantile \\
\hline No action & 75.8 & 39.8 & 140.2 \\
Immediate action & 104.2 & 89.1 & 143.7 \\
Optimal strategy & 61.6 & 39.3 & 121.5 \\
\hline
\end{tabular}

considered for decision making with regard to managing risks, similar to the concept of Value at Risk used in managing financial risks. If the optimal critical total damage level of USD 61.6 billion is just acceptable to the global environment and economy, there is about $30 \%$ chance this total damage will be exceeded and disastrous consequences may result. The $90 \%$ quantile of the total damage is USD 121.5 billion, twice as large as the expected value. In other words, there is $10 \%$ chance the total damage could reach twice as high as the acceptable level, which may be a disastrous outcome if this strategy is taken.

To see the impact on non-optimal stopping rule, we find distributions of $D(t, \tau)$ when stopping rule corresponds to different breaching levels $H=\omega S^{*}$ with $\omega$ ranging around 1 . For example, at $H=2.0$, the expected total damage obtained from Monte Carlo simulation is about 74. The corresponding critical temperature at which the action is taken is now 15.36 degrees, and the corresponding expected waiting time is 36 years. Comparing with the optimal strategy, this much earlier action at a much lower critical level of damage rate causes more total damage! At $H=1.0$, i.e. if we bite the bullet now without any waiting, the expected total damage obtained from Monte Carlo simulation is 104.2 (the exact value is 104.6), which is even larger than the damage of waiting for 36 years with $H=2.0$. Such is the paradox of the current model with these specific inputs. Finally, if no action is taken (the bullet is never bit), the expected total damage is 77.4 which is even better than taking action immediately. This is because the reduction in the damage rate due to immediate action is not large enough to justify the cost. The pdf curves of damage for the cases of no action and immediate action are also shown in Figure 1. The corresponding quantiles from Monte Carlo simulations for these three distributions are shown in Table 1.

In general, under the above inputs, any earlier (later) action or equivalently action at a lower (higher) threshold of the damage rate will be worse off than the optimal strategy. Intuitively, this does not seem to be expected: an earlier action does not reduce the total damage of global warming. This paradox can be explained by comparing the cost with the accumulated damage. The assumption of constant future cost means the present value of the cost decreases exponentially with time at the discount rate, and if this decrease is faster than the 
increase in the total damage in absolute terms, it is worthwhile to wait for some time before taking the action when the cost is much cheaper.

In the scenarios considered above, even the optimal strategy may not be good enough because of the high risk of too large a damage to the global economy. Assuming the process and especially the damage are correctly modeled, the only way out for confidently saving the global economy is to take more drastic measures to reduce the damage rate more aggressively with reduced cost. This means much reduced drift rate $\alpha_{2}$ after the bullet is bit, and at the same time keep the cost $K_{t}$ sufficiently low to enable effective and confident reduction of the total damage under the optimal strategy. This can be achieved through scientific research and technology breakthroughs.

The above paradox of earlier action being worse off than waiting disappears when we consider a more realistic scenario for the cost. Assuming the future cost increases with the discount rate, i.e. $q=r$, we then obtain a qualitatively different results. In this case, Monte Carlo simulation shows that the total damage becomes a monotonically increasing function of the breaching level. That is, the higher the critical value we take as the trigger of action, the larger the total damage will be. There is no optimal level in any delayed actions. The longer we wait, the larger will be the total damage. In this scenario the best approach is to take the action immediately.

For other cost increment rates $0<q<r$, the damage monotonically and continuously increases with the value of $q$. Figure 2 shows the damage curves corresponding to different values of $q$. For $q<r$ there is a minimum corresponding to the optimal policy. As the cost increasing rate $q$ approaches the economic growth rate $r=0.05$ from below, the damage curve converges to an asymptotic line. At $q \geq r$ the solution jumps to a different branch with optimal solution corresponding to an immediate action. This jump is easily confirmed by Monte Carlo simulation, see Fig. 2.

\section{CONCLUSIONS}

Finding optimal stopping rule via PDE approach is a very powerful method. In this paper the solution is found in closed form for $0<q<r$. Qualitatively, the optimal time to bite the bullet depends on, among other factors, the relative rates of economic growth and cost increment. For more realistic stochastic process for the damage rate and policy time dependence different from exponential, PDE can be solved numerically which is a subject of our future research.

\section{REFERENCES}

Association of American Geographyers (2003). Global change and local places: estimating, understanding, and reducing greehouse gases. Cambridge, UK: Cambridge University Press.

Conrad, J. M. (1997). Global warming: When to bite the bullet. Land Economics 2, 164-173.

Cox, D. R. and H. D. Miller (1965). The Theory of Stochastic Processes. London: Methuen.

Dixit, A. and R. Pindyck (1994). Investment under uncertainty. Princeton, NJ: Princeton University Press.

Field, C. B., V. Barros, T. F. Stocker, D. Qin, D. J. Dokken, K. L. Ebi, M. D. Mastrandreac, K. J. Mach, G. K. Plattner, S. K. Allen, M. Tignor, and P. M. M. (eds) (2012). Managing the Risks of Extreme Events and Disasters to Advance Climate Change Adaptation. A Special Report of Working Groups I and II of the Intergovernmental Panel on Climate Change. Cambridge, UK: Cambridge University Press.

Greiner, A., L. Grune, and W. Semmler (2010). Growth and climate change: threshold and multiple equilibria. Dynamic Modeling and Econometrics in Economics and Finance 12, 63-78.

Nordhaus, W. D. (1991). To slow or not to slow: the economics of the greenhouse effect. The Economic Journal 101, 920-937.

Nordhaus, W. D. (1992). An optimal transition path for controlling greenhouse gases. Science 258, 1315-1319.

Nordhaus, W. D. (2008). The question of balance. Hew Haven: Yale University Press.

Nordhaus, W. D. and J. Boyer (2000). Warming the world. Economic models of global warming. Cambridge: MIT Press.

UNFCCC (2006). Technologies for adaptation to climate change. Adaptation, Technology and Science Programme of United Nations Framework Convention on Climate Change Secretariat, Bonn, Germany, 40. 